\title{
Isometries and collineations of the Cayley surface
}

\author{
Johannes Gmainer \\ Hans Havlicek
}

full screen

close

quit

\begin{abstract}
Let $F$ be Cayley's ruled cubic surface in a projective three-space over any commutative field $K$. We determine all collineations fixing $F$, as a set, and all cubic forms defining $F$. For both problems the cases $|K|=2,3$ turn out to be exceptional. On the other hand, if $|K| \geq 4$ then the set of simple points of $F$ can be endowed with a non-symmetric distance function. We describe the corresponding circles, and we establish that each isometry extends to a unique projective collineation of the ambient space.
\end{abstract}

Keywords : Cayley surface, non-symmetric distance, isometry

MSC 2000: 51N25, 51N35, 51B15

\section{Introduction}

We investigate Cayley's ruled cubic surface $F$ in a three-dimensional projective space over an arbitrary commutative ground field $K$. It is fairly obvious that "most" of the results that are known from the classical case $(K=\mathbb{R}, \mathbb{C})$ will remain valid. However, a closer look shows that the situation is sometimes rather intricate.

In Section 3 we determine all collineations of the Cayley surface. If $|K| \leq 3$ then there are "more" such collineations than in the general case. From the proof of this result it is immediate that for $|K| \leq 3$ there are non-proportional cubic forms defining $F$. However, that proof does not answer the question of finding all such cubic forms to within a non-zero factor. We pay attention to this question, since it governs the interplay between incidence geometry and algebraic geometry. In Section 4 we show that the number of solutions to this problem equals 64 if $|K|=2$, two if $|K|=3$, and one otherwise. Our first 




Next, we assume $t$ to be variable, whence (18) gives

$$
\left(x_{22}-x_{11}^{2}\right) t^{2}+x_{21} t-\frac{x_{31}}{x_{11}}=0 \text { for all } t \in K^{\times} .
$$

According to the cardinality of $K$ there are three cases:

$|K|=2$. By (14), $x_{11}=x_{22}=x_{33}=1$ and (20) reads $x_{21} \cdot 1=x_{31}$, so that $\left(x_{i j}\right)=M_{0,0,1}$ or $\left(x_{i j}\right)=N$.

$|K|=3$. Then $x_{11}^{2}=1=t^{2}$ for $t \in\{1,2\}=K^{\times}$, and there are two possibilities: (i) $x_{22}=1$, whence (20) reads $x_{21} t-x_{31} / x_{11}=0$ for $t \in\{1,2\}$, so that $x_{21}=x_{31}=0$, and $\left(x_{i j}\right)=M_{0,0, c}$ with $c:=x_{11}$. (ii) $x_{22}=2$, whence (20) turns into $1 \cdot 1+x_{21} t-x_{31} / x_{11}=0$ for $t \in\{1,2\}$, so that $x_{21}=0$, $x_{31}=x_{11}$, and $\left(x_{i j}\right)=N_{c}$ with $c:=x_{11}$.

$|K| \geq 4$. From $\left|K^{\times}\right| \geq 3$ and (20) follows $x_{22}=x_{11}^{2}, x_{21}=x_{31}=0$, and $\left(x_{i j}\right)=M_{0,0, c}$ with $c:=x_{11}$.

In either case it is easy to see that the given matrices form a subgroup of $\mathrm{GL}_{4}(K)$.

We denote by $G_{\text {ext }}(F)$ the extended group of the Cayley surface, i.e. the group of all matrices $\left(x_{i j}\right)_{0 \leq i, j \leq 3} \in \mathrm{GL}_{4}(K)$, subject to the condition $x_{00}=1$, leaving invariant the Cayley surface $F$. By the first paragraph of the previous proof, each automorphic projective collineation of $F$ is induced by precisely one matrix in $G_{\text {ext }}(F)$. Furthermore, for $|K| \geq 4$, we have $G(F)=G_{\text {ext }}(F)$, whereas for $|K| \leq 3$ the groups $G(F)$ and $G_{\text {ext }}(F)$ are distinct, since none of the matrices $N$ and $N_{c}$ is in $G(F)$. We are thus lead to the following result:

Proposition 3.6. Let

$$
f_{(|K|)}(\boldsymbol{X}):= \begin{cases}X_{0} X_{1}^{2}+X_{0} X_{1} X_{2}+X_{1}^{3}+X_{0}^{2} X_{1}+X_{0}^{2} X_{3} & \text { when }|K|=2, \\ 2 X_{0} X_{1} X_{2}+2 X_{1}^{3}+2 X_{0}^{2} X_{1}+X_{0}^{2} X_{3} & \text { when }|K|=3 .\end{cases}
$$

Then, for $|K| \leq 3$, the Cayley surface $F=\mathcal{V}(f(\boldsymbol{X}))$ coincides with $\mathcal{V}\left(f_{(|K|)}(\boldsymbol{X})\right)$.

Proof. Let $|K|=2$. The image of $f(\boldsymbol{X})$ under the action of $N$ gives the polynomial $f_{(2)}(\boldsymbol{X})$. Likewise, for $|K|=3$, the polynomial $f_{(3)}(\boldsymbol{X})$, multiplied by $c \in\{1,2\}$, arises as the image of $f(\boldsymbol{X})$ under the action of $N_{c}$.

Observe that here "to coincide" just refers to sets of points and not to algebraic varieties in the sense of [8, p. 48]. Thus, for $|K| \leq 3$, the point set of the Cayley surface $F$ may also be considered as the algebraic curve $\mathcal{V}\left(f(\boldsymbol{X}), f_{|K|}(\boldsymbol{X})\right)$. 


\section{All cubic forms defining $F$}

In discussing the Cayley surface $F$ we have to distinguish between properties which stem from the defining polynomial $f(\boldsymbol{X})$ and geometric properties, i.e., properties which are invariant with respect to the action of the group $G_{\mathrm{ext}}(F)$. First, we recall some notions which can be defined in terms of $f(\boldsymbol{X})$. Let $\partial_{i}:=$ $\frac{\partial}{\partial X_{i}}$. We start by calculating the partial derivatives

$$
\begin{array}{llrl}
\partial_{0} f(\boldsymbol{X}) & =X_{1} X_{2}-2 X_{0} X_{3}, & & \partial_{1} f(\boldsymbol{X})=X_{0} X_{2}-3 X_{1}^{2}, \\
\partial_{2} f(\boldsymbol{X}) & =X_{0} X_{1}, & & \partial_{3} f(\boldsymbol{X})=-X_{0}^{2} .
\end{array}
$$

They vanish simultaneously at $\left(p_{0}, p_{1}, p_{2}, p_{3}\right)^{\mathrm{T}} \in K^{4 \times 1}$ if, and only if, at least one of the following conditions holds:

$$
\begin{gathered}
p_{0}=p_{1}=0 ; \\
p_{0}=p_{2}=0 \text { and char } K=3 .
\end{gathered}
$$

When $K$ is a field of characteristic char $K=3$ then, by (24), $\mathcal{V}\left(X_{0}, X_{2}\right)$ is a distinguished line in the ambient space of the Cayley surface $F$. Each of its points is a nucleus of $F$. See [8, p. 50] and [5, Proposition 3.17], where nuclei are defined in a slightly different way. All points subject to (23) are singular; they comprise the line $g_{\infty} \subset F$. We obtain, for all $s_{2}, s_{3} \in K$, that

$$
f\left(\left(0,0, s_{2}, s_{3}\right)+T \boldsymbol{X}\right)=T^{2} X_{0}\left(s_{2} X_{1}-s_{3} X_{0}\right)+T^{3}(*) \in K[\boldsymbol{X}, T] .
$$

Hence all points of $g_{\infty}$ are double points. The tangent cone (see [8, p. 49], where the term tangent space is used instead) at a point $Y=K\left(0,0, s_{2}, s_{3}\right)^{\mathrm{T}}$, $\left(s_{2}, s_{3}\right) \neq(0,0)$, is

$$
\mathcal{V}\left(X_{0}\left(s_{2} X_{1}-s_{3} X_{0}\right)\right)
$$

whence we refer to the plane at infinity as the tangent plane at $Y=Q_{3}$. For $Y=K\left(0,0,1, s_{3}\right)^{\mathrm{T}}$ the tangent cone is the union of the plane at infinity and the plane spanned by $g_{\infty}$ and the generator $g\left(1, s_{3}\right)$. We call each of these planes a tangent plane at $Y$. By (22), all points of $F \backslash g_{\infty}$ are simple. The tangent plane at $P\left(u_{1}, u_{2}\right)$ (see (3)) equals

$$
\mathcal{V}\left(\left(2 u_{1}^{3}-u_{1} u_{2}\right) X_{0}+\left(-3 u_{1}^{2}+u_{2}\right) X_{1}+u_{1} X_{2}-X_{3}\right) .
$$

Next, we present a characterization of tangent planes:

Proposition 4.1. Let $\tau=\mathcal{V}\left(\sum_{i=0}^{3} a_{i} X_{i}\right)$, where $a_{i} \in K$, be a plane. Then the following assertions are equivalent.

(a) $\tau$ is a tangent plane of $F$ with respect to $f(\boldsymbol{X})$. 





\section{Isometries of the Cayley surface $F$}

We shall assume $|K| \geq 4$ throughout this section.

Two (possibly identical) points of $F \backslash g_{\infty}$ are said to be parallel if they are on a common generator of $F$. This parallelism is an equivalence relation; it will be denoted by $\|$.

Let $A=P\left(u_{1}, u_{2}\right)$ and $B=P\left(v_{1}, v_{2}\right)$, where $u_{1}, u_{2}, v_{1}, v_{2} \in K$, be nonparallel points of $F \backslash g_{\infty}$. Thus $u_{1} \neq v_{1}$. The points of intersection of the line $A B$ and $F$ are in one-one correspondence with the zeros in $K$ of the polynomial

$$
\begin{aligned}
& f\left((1-T)\left(1, u_{1}, u_{2}, u_{1} u_{2}-u_{1}^{3}\right)+T\left(1, v_{1}, v_{2}, v_{1} v_{2}-v_{1}^{3}\right)\right) \\
& \quad=T(T-1)\left(u_{1}-v_{1}\right)\left(\left(u_{1}-v_{1}\right)^{2} T-2 u_{1}^{2}+u_{2}+u_{1} v_{1}-v_{2}+v_{1}^{2}\right) \in K[T],
\end{aligned}
$$

taking into account multiplicities. We read off that those zeros are 0,1 , and

$$
\delta(A, B):=\frac{2 u_{1}^{2}-u_{2}-u_{1} v_{1}+v_{2}-v_{1}^{2}}{\left(u_{1}-v_{1}\right)^{2}} .
$$

So $A B \cap F=\{A, B, C\}$ where, in terms of a cross ratio (CR), the point $C$ is given by

$$
\mathrm{CR}(C, B, A, I)=\delta(A, B) \text { with }\{I\}:=A B \cap \omega .
$$

If $A B \cap F=\{A, B\}$, i.e. when $\delta(A, B) \in\{0,1\}$, our definition of $C$ is based upon the intersection multiplicity of $A B$ at $A$ and $B$. This can be avoided as follows: By the last remark of the previous section, it is possible to decide in a purely geometric way whether $A B$ lies in the tangent plane of $F$ at $A$, whence $C=A$, or at $B$, whence $C=B$. (For this reason we adopted the assumption $|K| \geq 4$.)

Moreover, we define $\delta(A, B)=\infty$ whenever $A \| B$. So we are in a position to regard $\delta$ as a distance function

$$
\delta:\left(F \backslash g_{\infty}\right) \times\left(F \backslash g_{\infty}\right) \rightarrow K \cup\{\infty\} .
$$

It turns the affine part of the Cayley surface into a distance space in the sense of W. BENZ [1, p. 33]. We obtain

$$
\delta(A, A)=\infty \text { and } \delta(A, B)=1-\delta(B, A) \text { for all } A, B \in F \backslash g_{\infty},
$$

provided that we set $1-\infty:=\infty$. This distance function can be found in a paper by H. BRAUnER [4, p. 115] for $K=\mathbb{R}$ in a slightly different form. In terms of our $\delta$, Brauner's distance function can be expressed as

$$
\widehat{\delta}(A, B):=\frac{3}{2}\left(\frac{1}{2}-\delta(A, B)\right)^{-1} ;
$$




Theorem 5.4. The matrix group $G(F)$ has the following properties:

(a) $G(F)$ acts on $F \backslash g_{\infty}$ as a group of isometries.

(b) $G(F)$ acts regularly on the set of antiflags of $F \backslash g_{\infty}$.

(c) For each $d \in K$ the group $G(F)$ acts regularly on the set

$$
\Delta_{d}:=\left\{(A, B) \in\left(F \backslash g_{\infty}\right)^{2} \mid \delta(A, B)=d\right\} .
$$

(d) Let $A \| B$ and $A^{\prime} \| B^{\prime}$ be points of $F \backslash g_{\infty}$. Write $A=P\left(u_{1}, u_{2}\right), B=$ $P\left(u_{1}, v_{2}\right), A^{\prime}=P\left(u_{1}^{\prime}, u_{2}^{\prime}\right)$, and $B^{\prime}=P\left(u_{1}^{\prime}, v_{2}^{\prime}\right)$ with $u_{1}, u_{2}, \ldots, v_{2}^{\prime} \in K$. Then the number of matrices in $G(F)$ mapping $(A, B)$ to $\left(A^{\prime}, B^{\prime}\right)$ equals the number of distinct elements $c \in K^{\times}$such that

$$
c^{2}\left(v_{2}-u_{2}\right)=\left(v_{2}^{\prime}-u_{2}^{\prime}\right)
$$

Proof. (a) Let $A, B \in F \backslash g_{\infty}$. Suppose that $M \in G(F)$ takes $A$ to $A^{\prime}$ and $B$ to $B^{\prime}$. If $\delta(A, B) \neq 0,1, \infty$ then the line $A B$ meets the Cayley surface at three distinct points $A, B$, and $C$, say. Since $M$ preserves cross ratios, $\delta\left(A^{\prime}, B^{\prime}\right)=\delta(A, B)$ is immediate from (32). If $\delta=0$ then $A B$ is a tangent of $F$ at $A$. By the remark below (32), this tangent is mapped to a tangent of $F$ at $A^{\prime}$, whence $\delta\left(A^{\prime}, B^{\prime}\right)=0$, as required. The case $\delta(A, B)=1$ can be treated similarly. Finally, $\delta(A, B)=\infty$ means that $A, B$ are on a common generator, a property which is shared by their images, whence the assertion follows.

(b) Since $G(F)$ acts transitively on $F \backslash g_{\infty}$, it is sufficient to show that the stabilizer of $Q_{0}$ in $G(F)$, i.e. $\left\{M_{0,0, c} \mid c \in K^{\times}\right\}$, acts regularly on $\{g(1, c) \mid$ $\left.c \in K^{\times}\right\}$. In fact, if we are given generators $g\left(1, c_{1}\right)$ and $g\left(1, c_{2}\right)$ with $c_{1}, c_{2} \in K^{\times}$then $M_{0,0, c_{2} c_{1}^{-1}}$ is the only solution.

(c) Let $(A, B)$ and $\left(A^{\prime}, B^{\prime}\right)$ be elements of $\Delta_{d}$. By Lemma 3.3, we may assume w.l.o.g. that $A=A^{\prime}=P(0,0)$. We infer from (31) that a point $Y=$ $P\left(y_{1}, y_{2}\right), y_{1}, y_{2} \in K$, satisfies $\delta(A, Y)=d$ if, and only if, $y_{2}=(d+1) y_{1}^{2}$ and $y_{1} \in K^{\times}$. So there exist elements $u_{1}, u_{1}^{\prime} \in K^{\times}$with

$$
B=P\left(u_{1},(d+1) u_{1}^{2}\right), B^{\prime}=P\left(u_{1}^{\prime},(d+1) u_{1}^{\prime 2}\right) .
$$

Letting $c:=u_{1}^{\prime} u_{1}^{-1}$, the matrix $M_{0,0, c}$ has the required property. The point $A$ and the unique generator through $B$ form an antiflag; the same holds for $A^{\prime}$ and the unique generator through $B^{\prime}$. So the asserted regularity is a consequence of (b). 


